\title{
Partial differential matrix equations for the inverse problem of scattering theory
}

\author{
M. Coz ${ }^{*}$ and P. Rochus \\ Universite de Liège, Physique Nucléaire Théorique, Institut de Physique au Sart Tilman, B-4000 Liège \\ 1, Belgium \\ (Received 8 December 1975)
}

Sufficient conditions for the existence of a continuous translation operator are found in the case of a systern of differential equations in which the matrix potential has the singularity of the centripetal term. The sufficient conditions are found in terms of moments of the nuclear potential. The method used employs the Riemann Green's function. Threshold energies introduce a threshold energy dependence into the translation kernel and lead to a requirement of an exponential decrease for terms of the matrix potential.

\section{INTRODUCTION}

The partial differential equations for the inverse problem of scattering have received considerable attention since Agranovitch and Marchenko's excellent mono$\operatorname{graph}^{1}$ (A and M) appeared. Leaving aside the onedimensional problem, ${ }^{2}$ authors who have followed $\mathrm{A}$ and $M$ have limited their concern to scalar potentials. ${ }^{3-6}$ By so doing they lost the purpose of Ref. 1, where explicitly matrix differential equations were studied. However, the primary concern of Ref. 1 was not differential equations as such; after the results for the nonsingular case were obtained, they considered an indirect approach to the singular case via the transformation techniques of Crum and Krein. ${ }^{7,8}$

Since the work of Gugushvili and Mentkovsky ${ }^{5}$ and $\mathrm{Coz}$ and Coudray, ${ }^{6}$ it is possible to study directly systems of differential equations where the singularity comes from an explicit dependence on the angular momentum. In the present paper, we consider finite systems of $n$ differential equations:

$$
\left[L(x)+\Lambda^{2}\right] u(\Lambda, x)=V u(\Lambda, x),
$$

where $L, \Lambda, V, u$ are $(n \times n)$ matrices. The columns of $u(\Lambda, x)$ are solutions of Eq. (1) which differ from each other by their boundary conditions. $\Lambda$ is the diagonal matrix of the channel wavenumbers $\lambda_{1}, \ldots, \lambda_{n}$ :

$$
\Lambda_{i j} \equiv \lambda_{i} \delta_{i j},
$$

$V$ is an Hermitian matrix potential which is called the nuclear potential; finally, the elements of the differential operator $L(x)$ are

$$
\begin{aligned}
& L(x)=D(x)-l(x), \\
& D_{i j}(x) \equiv \frac{d^{2}}{d x^{2}} \delta_{i j}, \quad l_{i j}(x) \equiv \frac{l_{i}\left(l_{i}+1\right)}{x^{2}} \delta_{i j} .
\end{aligned}
$$

The solution we are concerned with is the solution which is singular at the origin and defined by its behavior at infinity. Our choice of solution, that of Ref. 1, is dictated by the relationship of this solution to the $S$ matrix. The matrix solution $H(\Lambda, x)$ for the homogeneous system is the Riccati Hankel function

$$
\begin{aligned}
H_{i j}(\Lambda, x)=-i x h_{l_{i}}^{(2)}\left(\lambda_{i} x\right) \delta_{i j} & =-i \sqrt{\pi x} / 2 H_{i^{+1 / 2}}^{(2)}\left(\lambda_{i} x\right) \delta_{i j} \\
& =(-)^{l_{i}} H_{i j}(-\Lambda, x) .
\end{aligned}
$$

The Green's function for the homogeneous system is the diagonal $G$ function matrix:

$$
\begin{aligned}
G_{i j}(\Lambda ; x, y)= & 0 \text { for } y \leqslant x \\
G_{i j}(\Lambda ; x, y)= & i(-)^{l_{i}}\left(2 \lambda_{i}\right)^{-1}\left[h_{i_{i}}^{(2)}\left(\lambda_{i} x\right) h_{i_{i}}^{(2)}\left(-\lambda_{i} y\right)\right. \\
& \left.-h_{l_{i}}^{(2)}\left(-\lambda_{i} x\right) h_{i_{i}}^{(2)}\left(\lambda_{i} y\right)\right] \delta_{i j} \text { for } x \leqslant y .
\end{aligned}
$$

The irregular matrix solution $F(\Lambda, x)$ is then defined by

$$
F(\Lambda, x)=H(\Lambda, x)-\int_{x}^{\infty} G(\Lambda, x, y) V(y) F(\Lambda, y) d y,
$$

where its boundary conditions have been incorporated. We consider the possible integral representation for $F(\Lambda, x)$ :

$$
F(\Lambda, x)=H(\Lambda, x)+\int_{x}^{\infty} K(x, y) H(\Lambda, y) d y 。
$$

The case where all $\lambda_{i}$ are equal is studied first. Then no dependence on the threshold energies appears in the translation kernel $K(x, y)$ of $\mathrm{Eq}$. (7). The general case is investigated in Sec. 4. Sufficient conditions for the matrix $K$ to exist are the goal of this paper.

The kernel $K$ is connected with the solution of the inverse problem as is seen from Eq. (9) below. It satisfies the matrix partial differential equation

$$
\begin{aligned}
& {[D(x)-D(y)] K(x, y)-[l(x) K(x, y)-K(x, y) l(y)]} \\
& \quad=V(x) K(x, y) .
\end{aligned}
$$

The boundary conditions for Eq. (8) are

$$
\begin{aligned}
& K(x, x)=\frac{1}{2} \int_{x}^{\infty} V(s) d s, \\
& \lim _{y \rightarrow \infty} K(x, y)=\lim _{y \rightarrow \infty} \frac{\partial}{\partial y} K(x, y)=0 .
\end{aligned}
$$

The development of Eq. (8) with its conditions (9) and (10) is sketched in Appendix A. The same type of equation is found in Ref. 9. The kernel $K$ is therefore connected with the resolvent of $\mathrm{Eq}$. (6). The equation defining $K$ belongs to the hyperbolic type and our work is dependent upon results for these equations in Refs. 10 and 11, which we extend here beyond scalar interactions.

\section{THE RIEMANN'S SOLUTION AND NOTATIONS}

Equation (8) is rewritten explicitly 


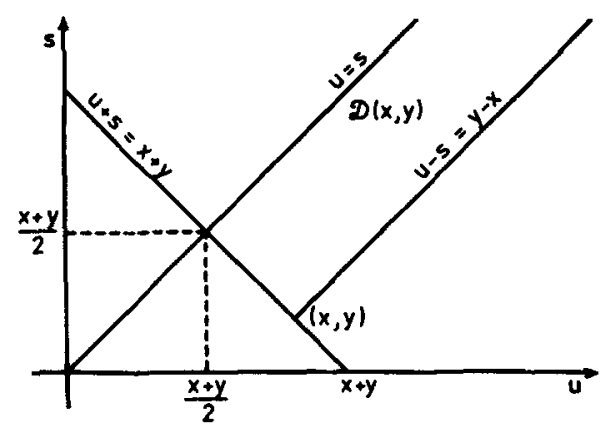

FIG. 1 .

$$
\begin{aligned}
\left(\frac{d^{2}}{d x^{2}}-\frac{l_{i}\left(l_{i}+1\right)}{x^{2}}\right) K_{i j}(x, y)= & \left(\frac{d^{2}}{d y^{2}}-\frac{l_{j}\left(l_{j}+1\right)}{y^{2}}\right) K_{i j}(x, y) \\
& +\sum_{k} V_{i k}(x) K_{k j}(x, y)
\end{aligned}
$$

It is solved using Riemann's method, which is recalled now. ${ }^{12}$ A Riemann solution $R(x, y ; s, u)$ is defined. It satisfies

$$
\begin{aligned}
& \left(\frac{d^{2}}{d x^{2}}-\frac{l_{i}\left(l_{i}+1\right)}{x^{2}}\right) R_{i j}(x, y ; s, u) \\
& =\left(\frac{d^{2}}{d y^{2}}-\frac{l_{j}\left(l_{j}+1\right)}{y^{2}}\right) R_{i j}(x, y ; s, u), \\
& R_{i j}(x, y ; x, y)=1, \\
& \frac{\partial}{\partial x} R+\frac{\partial}{\partial y} R=0 \text { if } y-x=u-s, \\
& \frac{\partial}{\partial x} R-\frac{\partial}{\partial y} R=0 \text { if } y+x=u+s
\end{aligned}
$$

With the use of characteristic curves, Eq. (11) with its boundary conditions is solved by the Riemann's method, and one has

$$
\begin{aligned}
K_{i j}(x, y)= & \frac{1}{2} \int_{(x+y) / 2}^{\infty} R_{i j}(x, y ; s, s) V_{i j}(s) d s \\
& +\frac{1}{2} \int_{x}^{(x+y) / 2} d s \int_{y+x-s}^{y+s-x} R_{i j}(x, y ; s, u) \\
& \times \sum_{k} V_{i k}(s) K_{k j}(s, u) d u \\
& +\frac{1}{2} \int_{(x+y) / 2}^{\infty} d s \int_{s}^{y+s-x} R_{i j}(x, y ; s, u) \\
& \times \sum_{k} V_{i k}(s) K_{k j}(s, u) d u, \quad 0<x \leqslant y .
\end{aligned}
$$

We will shorten our notations by writing

$$
\begin{aligned}
K_{i j}(x, y)= & \frac{1}{2} \int_{(x+y) / 2}^{\infty} R_{i j}(x, y ; s, s) V_{i j}(s) d s \\
& +\frac{1}{2} \iint_{(x, y)} R_{i j}(x, y ; s, u) \\
& \times \sum_{k} V_{i k}(s) K_{k j}^{*}(s, u) d u d s,
\end{aligned}
$$

with $d u$ preceding $d s$ in the double integral. $D(x, y)$ is the integration domain of Fig. 1.

Together with Riemann's solution, we shall introduce, for each matrix $A$, the matrix of its absolute values $|A|$ whose elements are

$$
|A|_{i j}=\left|A_{i j}\right|
$$

If $A$ and $B$ are matrices, we will write $A \leqslant B$ if

$$
\left|A_{i j}\right| \leqslant\left|B_{i j}\right| \text { for all } i, j \leqslant n \text {. }
$$

Clearly, Eq. (18) implies

$$
|A B C| \leqslant|A||B||C| \text {. }
$$

For each matrix $A$, we will also define a norm $\|A\|$ by

$$
\|A\|=\max _{j} \sum_{k}\left|A_{j k}\right|,
$$

Clearly, $\|A\|$ introduced by Eq. (20) has the usual properties of a norm. ${ }^{13}$

\section{A PARTICULAR CASE}

A special case is studied now since it allows us to use standard matrix methods and to indicate how results can be obtained. It contains an extension of Marchenko's results.

This special case is obtained by writing $[D(x)-l(x)] K(x, y)=[D(y)-l(y)] K(x, y)=V(x) K(x, y)$. To be more specific, Eq. (11) is replaced by $\mathrm{Eq} .(20)$ :

$$
\begin{aligned}
\left(\frac{d^{2}}{d x^{2}}-\frac{l_{i}\left(l_{i}+1\right)}{x^{2}}\right) K_{i j}(x, y)= & \left(\frac{d^{2}}{d y^{2}}-\frac{l_{i}\left(l_{i}+1\right)}{y^{2}}\right) K_{i j}(x, y) \\
& +\sum_{k} V_{i k}(x) K_{k j}(x, y) .
\end{aligned}
$$

However, the boundary conditions which $K$ should satisfy, i. e., Eqs. (9) and (10), are retained.

The Riemann functions to be used are the solutions of

$$
\begin{aligned}
& \left(\frac{d^{2}}{d x^{2}}-\frac{l_{i}\left(l_{i}+1\right)}{x^{2}}\right) R_{i i}(x, y ; s, u) \\
& \quad=\left(\frac{d^{2}}{d y^{2}}-\frac{l_{i}\left(l_{i}+1\right)}{y^{2}}\right) R_{i i}(x, y ; s, u) .
\end{aligned}
$$

We can define a Riemann matrix by

$$
[D(x)-l(x)] R(x, y ; s, u)=[D(y)-l(y)] R(x, y ; s, u) \text {. }
$$

Using Chaundy's results, Ref. 9, one can write

$$
R(x, y ; s, u)=\left\{P_{l_{i}}(1-2 z)\right\} \delta_{i j}
$$

with

$$
\begin{aligned}
z= & (1 / 16 u s x y)(x+y-u-s)(s-u+y-x)(u+y+s+x) \\
& \times(u+y-s-x),
\end{aligned}
$$

as illustrated in Ref. 6. Instead of $z$ we use Chaundy's variables $x_{1}$ and $x_{2}$ defined as follows

$$
\begin{aligned}
& x_{1}=\frac{(u+s-x-y)(x-z-s+u)}{4 x s}, \\
& x_{2}=\frac{(x+y-u-s)(x-y+u-s)}{4 u y}, \\
& 1-2 z=1-2 x_{1}-2 x_{2}+2 x_{1} x_{2},
\end{aligned}
$$

and write

$$
R(x, y ; u, s)=\left\{P_{t_{i}}\left(1-2 x_{1}-2 x_{2}+2 x_{1} x_{2}\right) \delta_{i j}\right\} .
$$

In the Domain $\nu(x, y)$, in Fig. 1, the following inequalities are satisfied:

$$
u-s \leqslant y-x,
$$




$$
\begin{aligned}
& u+s \geqslant y+x, \\
& u \geqslant s, \\
& s \geqslant x, \\
& y \geqslant x .
\end{aligned}
$$

From these inequalities the following estimates can be derived:

$$
\begin{array}{ll}
u+s-x-y \leqslant 2(s-x) \leqslant 2 s & \text { by }(24), \\
s-u+y-x \leqslant 2(s-x) \leqslant 2 s & \text { by }(24), \\
u+y+s+x \leqslant 2(u+s) \leqslant 4 u & \text { by }(25) \text { and }(26), \\
u+y-s-x \leqslant 2(y-x) \leqslant 2 y & \text { by (24). }
\end{array}
$$

While $x_{1}$ is negative and

$$
-x_{1} \leqslant s / x \text {, }
$$

$x_{2}$ is positive

$$
x_{2}=\frac{(u+s-x-y)(y-x+s-u)}{4 u y} \leqslant \frac{(u+s)(u+y-s-x)}{4 u y},
$$

since one has

$$
(u+y-s-x)-(y-x-s-u)=2(u-s) \geqslant 0 .
$$

Therefore, one obtains

$$
0 \leqslant x_{2} \leqslant 1 \text {. }
$$

We rewrite the argument

$$
z=\left(1-2 x_{1}\right)-2 x_{2}\left(1-x_{1}\right)
$$

with the two parentheses positive. Equations (29) and (30) imply

$$
1 \leqslant x \leqslant 1-2 x_{1} \leqslant 3 s / x \text {. }
$$

The argument $X$ of the Legendre functions being greater than unity, one has

$$
\left|P_{l}(X)\right| \leqslant(2 X)^{l} \leqslant(6 s / x)^{l} .
$$

Consequently,

$$
|R(x, y ; s, u)| \leqslant D^{-1}(x) C D(s)
$$

where the diagonal matrices $D$ and $C$ have the following elements:

$$
\begin{aligned}
& C_{i j}=6^{l_{i}} \delta_{i j}, \\
& D_{i j}(s)=s^{l_{i}} \delta_{i j} .
\end{aligned}
$$

The integral equation for the matrix $K$ can be written in a matrix form:

$$
\begin{aligned}
K(x, y)= & \frac{1}{2} \int_{(x+y) / 2}^{\infty} R(x, y, ; s, s) V(s) d s \\
& +\frac{1}{2} \iint_{\partial_{(x, y)}} R(x, y ; s, u) V(s) K(s, u) d u d s .
\end{aligned}
$$

We use now the reduction method of Ref. $6 b$ and define

$$
\begin{aligned}
& K(x, y)=D^{-1}(x) \tilde{K}(x, y), \\
& R(x, y ; s, u)=D^{-1}(x) \tilde{R}(x, y ; s, u) D(s) C
\end{aligned}
$$

with

$$
|R| \leqslant I \text { (matrix unity). }
$$

With these reductions Eq. (37) becomes

$$
\begin{aligned}
\tilde{K}(x, y)= & =\frac{1}{2} \int_{(x+y) / 2}^{\infty} \tilde{R}(x, y ; s, s) D(s) C V(s) d s \\
& +\frac{1}{2} \iint_{D(x, y)} \tilde{R}(x, y ; s, u) D(s) \\
& \times C V(s) D^{-1}(s) \tilde{K}(s, u) d u d s .
\end{aligned}
$$

We are led to define the two interactions

$$
\begin{aligned}
& \tilde{V}(s)=D(s) C V(s), \\
& W(s)=D(s) C V(s) D^{-1}(s),
\end{aligned}
$$

which should allow us to use the estimates of Appendix B. We decide here to proceed otherwise for a better interpretation of the conditions we obtain.

Equation (41) is rewritten as

$$
\begin{aligned}
\tilde{K}(x, y)= & \frac{1}{2} \int_{(x+y) / 2}^{\infty} \tilde{R}(x, y ; s, s) \tilde{V}(s) d s \\
& +\iint_{D(x, y)} \tilde{R}(x, y ; s, u) W(s) \tilde{K}(s, u) d u d s
\end{aligned}
$$

and solved by the method of successive approximations:

$$
\tilde{K}(x, y)=\sum_{n=0}^{\infty} \tilde{K}^{(n)}(x, y),
$$

where

$$
\tilde{K}^{(0)}(x, y)=\frac{1}{2} \int_{(x+y) / 2}^{\infty} \tilde{R}(x, y ; s, s) \tilde{V}(s) d s
$$

and

$$
\begin{aligned}
\tilde{K}^{(n)}(x, y)= & \frac{1}{2} \iint_{D(x, y)} \tilde{R}(x, y ; s, u) W(s) \\
& \times \tilde{K}^{(n-1)}(s, u) d u d s .
\end{aligned}
$$

The following bounds are found for the different terms of the series defined in Eq. (43):

$$
\begin{aligned}
\left|\tilde{K}^{(0)}(x, y)\right| \leqslant & \frac{1}{2} \int_{(x+y) / 2}^{\infty}|\tilde{V}(s)| d s \equiv \frac{1}{2} \sigma((x+y) / 2), \\
\left|\tilde{K}^{(1)}(x, y)\right| \leqslant & \frac{1}{2} \iint_{\mathcal{D}(x, y)}|\tilde{R}(x, y ; s, u)||W(s)| \\
& \times\left|\tilde{K}^{(0)}(s, u)\right| d u d s \\
& \leqslant \frac{1}{2} \int_{x}^{\infty}|W(s)| s d s \sigma((x+y) / 2) .
\end{aligned}
$$

More generally one has

$$
\begin{aligned}
& \left|\tilde{K}^{(n)}(x, y)\right| \\
& \quad \leqslant \frac{1}{2} \int_{x}^{\infty} d x_{n-1} x_{n-1}\left|W\left(x_{n-1}\right)\right| \int_{x_{n-1}}^{\infty} d x_{n-2} x_{n-2}\left|W\left(x_{n-2}\right)\right| \\
& \quad \times \cdots \int_{x_{1}}^{\infty} d x_{0} x_{0}\left|W\left(x_{0}\right)\right| \sigma((x+y) / 2) .
\end{aligned}
$$

We determine now necessary and sufficient conditions for a convergence of the series (43) which is both uniform and absolute. These conditions, however, are simply sufficient conditions for the existence of $\tilde{K}$. For the series (43) to converge uniformly and absolutely it is necessary to impose

$$
\begin{aligned}
& \int_{x}^{\infty}|\tilde{V}(s)| d s<\infty, \\
& \int_{x}^{\infty}|W(s)| s d s<\infty .
\end{aligned}
$$


The potential $V_{i j}$ should have a moment of order $l_{i}$ from Eq. (49) and a moment of order $\left(l_{i}-l_{j}+1\right)$ from Eq. (50).

To prove the sufficiency of these conditions, norms are used as in Appendix B. With Eqs. (49) and (50) one can write

$$
\|\tilde{K}(x, y)\| \leqslant \frac{1}{2} \eta((x+y) / 2) \exp [\xi(x)]
$$

with

$$
\begin{aligned}
& \eta((x+y) / 2)=\int_{(x+y) / 2}^{\infty}\|V(s)\| d s \\
& \xi(x)=\int_{x}^{\infty}\|W(s)\| d s .
\end{aligned}
$$

Equation (51) defines an estimate for the norm of $K(x, y)$

$$
\|K(x, y)\|=D^{-1}(x)\|\tilde{K}(x, y)\| .
$$

From Eq. (54) it can be seen that the matrix $K(x, y)$ when $\tilde{K}$ satisfies Eq. (51) is a matrix of absolutely integrable functions with respect to $y$.

A physical application of this section is that when all the $l_{i}$ are equal

$$
l_{i}=l \text {. }
$$

Equation (55) contains as a particular case Marchenko's study where all the $l_{i}=0$. From what precedes, moments of order $l$ and one should be required from the elements of the matrix potential. So we state:

Theorem: If

$$
\begin{aligned}
& \sigma_{1}(x)=\int_{x}^{\infty} t|V(t)| d t<\infty, \\
& \sigma_{l}(x)=\int_{x}^{\infty} t^{l}|V(t)| d t<\infty,
\end{aligned}
$$

then an integral representation ${ }^{7}$ exists for the solution of a partial differential matrix equation with all $l$ equal, and one has

$$
(1 / x)^{l}\|K(x, y)\| \leqslant \frac{1}{2} \sigma_{l}((x+y) / 2) \exp \left[\sigma_{1}(x)\right]
$$

\section{THE GENERAL CASE EQ (11)}

To follow the same method, we need to use the Riemann's solutions of Eq. (12). These two can be found in Refs. 10 and 11:

$$
\begin{aligned}
R_{i j}(x, y ; s, u) & =\pi\left[\begin{array}{l}
l_{i}, l_{j} \\
x_{1}, x_{2}
\end{array}\right] \\
\varkappa\left[\begin{array}{l}
l_{i}, l_{j} \\
x_{1}, x_{2}
\end{array}\right]= & P_{i_{i}}\left(1-2 x_{1}\right) \\
& -2 x_{2} \int_{0}^{1} P_{l_{i}}\left(1-2 x_{1}+2 x_{1} t\right) P_{t_{j}}^{\prime}\left(1-2 x_{2} t\right) d t,
\end{aligned}
$$

where $x$ is a Chinese letter, to be pronounced "pa," introduced by Chaundy (Ref. 10).

Equation (56) is integrated by parts to get

$R_{i j}=P_{i_{j}}\left(1-2 x_{2}\right)-2 x_{1} \int_{0}^{1} P_{l_{j}}\left(1-2 x_{2} t\right) P_{i_{i}}^{\prime}\left(1-2 x_{1}+2 x_{1} t\right) d t$.

Now we consider

$$
\begin{aligned}
I & =\left|(-1) \int_{0}^{1} P_{l_{j}}\left(1-2 x_{2} t\right) 2 x_{1} P_{l_{i}}^{\prime}\left(1-2 x_{1}+2 x_{1} t\right) d t\right| \\
& \leqslant \int_{0}^{1} \mid\left[-2 x_{1} P_{l_{1}}^{\prime}\left(1-2 x_{1}+2 x_{1} t\right) \mid d t .\right.
\end{aligned}
$$

To get Eq. (57), we used the fact that $\left|1-2 x_{2} t\right| \leqslant 1$ implied

$$
\left|P_{t_{j}}\left(1-2 x_{2} t\right)\right| \leqslant 1
$$

The integrand in Eq. (57) has a constant sign since $P_{l_{1}}$ is an increasing function of its argument whenever the latter is greater than unity.

Consequently,

$$
\begin{aligned}
I & \leqslant\left|\int_{0}^{1}\left(-2 x_{1}\right) P_{i_{1}}^{\prime}\left(1-2 x_{1}+2 x_{1} t\right)\right| \\
& \leqslant P_{t_{i}}\left(1-2 x_{1}\right) .
\end{aligned}
$$

The estimate for $R_{i j}$ follows:

$$
\left|R_{i j}\right| \leqslant(6 s / x)^{l_{i}}+1 \leqslant(7 s / x)^{i_{i}} \text {. }
$$

Back to Eq. (17), we use again reductions

$$
\left\{\begin{array}{l}
\left(1 / x^{l_{i}}\right) K_{i j}=\tilde{K}_{i j}, \\
R_{i j}=\left(1 / x^{i}\right) \tilde{R}_{i j}(7 s)^{l_{i}}, \quad\left|\tilde{R}_{i j}\right|<1, \\
\tilde{V}_{i j}=\left(7 s^{l_{i}}\right) V_{i j}, \\
W_{i j}=\left(7 s^{l_{i}}\right) V_{i j} / x^{l_{j}} .
\end{array}\right.
$$

Thus we write Eq. (17) as

$$
\begin{aligned}
\tilde{K}_{i j}(x, y) & =\frac{1}{2} \int_{(x+y) / 2}^{\infty} \tilde{R}_{i j}(x, y ; s, s) \tilde{V}_{i j}(s) d s \\
& +\frac{1}{2} \iint_{D_{(x, y)}} \tilde{R}_{i j}(x, y ; s, u) \\
& \times \sum_{k} W_{i k}(s) \tilde{K}_{k j}(s, u) d s d u .
\end{aligned}
$$

So we will get

$$
\begin{aligned}
& \left|\tilde{K}_{i j}^{(0)}(x, y)\right| \leqslant \frac{1}{2} \int_{(x+y) / 2}^{\infty}\left|\tilde{V}_{i j}(s)\right| d s, \\
& \left|\tilde{K}_{i j}^{(1)}(x, y)\right| \leqslant \frac{1}{2} \int_{x}^{\infty}|s| \sum_{k}\left|W_{i k}(s)\right|\left|\tilde{K}_{k j}^{(0)}(x, y)\right| d s .
\end{aligned}
$$

Equations (61) are similar in form to Eqs. (46) and (47) when the latter are made explicit in $i$ and $j$. The analysis of the previous section applies therefore, and we can state the theorem.

Theorem: If

$$
\begin{aligned}
& \sigma_{i j}^{(1)}(x)=\int_{x}^{\infty} t\left|V_{i j}(t)\right| t^{\left(i_{i}-l_{j}\right)} d t<\infty, \\
& \sigma_{i j}^{(0)}(x)=\int_{x}^{\infty} t^{i} i\left|V_{i j}(t)\right| d t<\infty,
\end{aligned}
$$

then an integral representation ${ }^{7}$ for the solution of the partial differential matrix equation exists, and one has

$$
D^{-1}(x)\|K(x, y)\| \leqslant \frac{1}{2} \eta((x+y) / 2) \exp [\xi(x)] .
$$

In Eq. (62) the definitions

$$
\eta((x+y) / 2)=\int_{(x+y) / 2}^{\infty}\|V(s)\| d s \quad \eta(x)=\int_{x}^{\infty}\|W(s)\| s d s
$$

were used with $\tilde{V}$ and $W$ taken from Eq. (59). 
We proceed now to remove the restriction that all the $\lambda_{i}$ 's should be equal. If they are not equal, the $K_{i j}$ elements of the $K$ matrix are the solutions of the equation

$$
\begin{aligned}
\left(\frac{\partial^{2}}{\partial x^{2}}+\lambda_{i}{ }^{2}-\frac{l_{i}\left(l_{i}+1\right)}{x^{2}}\right) K_{i j}(x, y) & \\
= & \left(\frac{\partial^{2}}{\partial y^{2}}+\lambda_{j}{ }^{2}-\frac{l_{j}\left(l_{j}+1\right)}{y^{2}}\right) K_{i j}(x, y) \\
& +\sum_{k} V_{i k}(x) K_{k j}(x, y),
\end{aligned}
$$

which introduces a $\lambda$ dependence in the $K$ matrix.

We need now the Riemann solution $X_{i j}$ for the partial differential equation:

$$
\begin{aligned}
& \left(\frac{\partial^{2}}{\partial x^{2}}+\lambda_{i}{ }^{2}-\frac{l_{i}\left(l_{i}+1\right)}{x^{2}}\right) X_{i j} \\
& =\left(\frac{\partial^{2}}{\partial y^{2}}+\lambda_{j}{ }^{2}-\frac{l_{j}\left(l_{j}+1\right)}{y^{2}}\right) X_{i j} .
\end{aligned}
$$

To obtain an estimate for these $X_{i j}$, Theorem 1 of Ref. $6 \mathrm{~b}$ is used. Using characteristic variables $\left(\xi, \eta, \xi_{0}, \eta_{0}\right)$ instead of the physical variables $(x, y ; s, u)$ and denoting

$$
\begin{aligned}
& X\left(\xi, \eta ; \xi_{0}, \eta_{0}\right) \equiv X(x, y ; s, u), \\
& R\left(\xi, \eta ; \xi_{0}, \eta_{0}\right) \equiv R(x, y ; s, u),
\end{aligned}
$$

we have

$$
\begin{aligned}
X_{i j}\left(\eta, \eta ; \xi_{0}, \eta_{0}\right)= & R_{i j}\left(\xi, \eta ; \xi_{0}, \eta_{0}\right)+\int_{\xi}^{\xi_{0}} d \xi^{\prime} \\
& \times \int_{\eta}^{\eta_{0}} d \eta^{\prime} R_{i j}\left(\xi, \eta ; \xi^{\prime}, \eta^{\prime}\right)\left(\lambda_{i}{ }^{2}-\lambda_{j}{ }^{2}\right) \\
& \times X_{i j}\left(\xi^{\prime}, \eta^{\prime}, \xi_{0}, \eta_{0}\right) .
\end{aligned}
$$

Since $\left|R_{i j}\right| \leqslant(7 s / x)^{l_{i}}$, we have

$$
\left|R_{i j}\right| \leqslant\left[7\left(\xi_{0}-\eta_{0}\right) /(\xi-\eta)\right]^{l_{i}} \text {. }
$$

We set

$$
\begin{aligned}
& R_{i j}=\tilde{R}_{i j}\left[7\left(\xi_{0}-\eta_{0}\right) /(\xi-\eta)\right]^{i} i, \\
& X_{i j}=\tilde{X}_{i j}\left[7\left(\xi_{0}-\eta_{0}\right) /(\xi-\eta)\right]^{i},
\end{aligned}
$$

and obtain the reduced equation for $\tilde{X}_{i j}$ :

$$
\begin{aligned}
\tilde{X}_{i j}(\xi, & \left.\eta ; \xi_{0}, \eta_{0}\right) \\
= & \tilde{R}_{i j}\left(\xi, \eta ; \xi_{0}, \eta_{0}\right)+\int_{\xi}^{\xi_{0}} d \xi^{\prime} \int_{\eta}^{\eta_{0} 0} d \eta^{\prime} \\
& \times \tilde{R}_{i j}\left(\xi, \eta ; \xi^{\prime}, \eta^{\prime}\right)\left(\lambda_{i}{ }^{2}-\lambda_{j}{ }^{2}\right) \tilde{X}_{i j}\left(\xi^{\prime}, \eta^{\prime} ; \xi_{0}, \eta_{0}\right) .
\end{aligned}
$$

From this Eq. (66) we get ${ }^{14}$

$$
\begin{aligned}
& \left|\tilde{X}_{i j}\left(\xi, \eta ; \xi_{0}, \eta_{0}\right)\right| \\
& \quad \leqslant \exp \left[2\left(\left|\lambda_{i}{ }^{2}-\lambda_{j}{ }^{2}\right|\left|\xi_{0}-\xi\right|\left|\eta_{0}-\eta\right|\right)^{1 / 2}\right] \\
& \left|\tilde{X}_{i j}(x, y ; s, u)\right| \\
& \quad \leqslant \exp \left[2\left(\left|\lambda_{i}{ }^{2}-\lambda_{j}{ }^{2}\right||u-s-y+x||u+s-y-x|\right)^{1 / 2}\right] .
\end{aligned}
$$

Now we have from Eq. (29)

$$
\begin{array}{ll}
u+s-x-y \geqslant 0, & u+s-y-x \leqslant 2 s, \\
u-s+x-y \leqslant 0, & s-u+y-x \leqslant 2 s .
\end{array}
$$

Setting

$$
\beta^{2}=\sup _{i, j}\left|\lambda_{i}^{2}-\lambda_{j}^{2}\right|
$$

we obtain the estimate

$$
\left|X_{i j}(x, y ; s, u)\right| \leqslant(7 s / x)^{l_{i}} \exp (4 \beta s),
$$

valid for all $i$ and all $j$.

Now we define

$$
\begin{aligned}
& \tilde{V}_{i j}(s)=(7 s)^{l_{i}} V_{i j}(s) \exp (4 \beta s), \\
& W_{i j}(s)=(7)^{l_{i}} s^{\left(i_{i}-l_{j}\right)} V_{i j}(s) \exp (4 \beta s) .
\end{aligned}
$$

The equation for $\tilde{K}_{i j}$ follows:

$$
\begin{aligned}
\tilde{K}_{i j}(x, y)= & \frac{1}{2} \int_{(x+y) / 2}^{\infty} \tilde{X}_{i j}(x, y ; s, s) \tilde{V}_{i j}(s) d s \\
& +\frac{1}{2} \iint_{D(x, y)} \tilde{X}_{i j}(x, y ; s, u) \sum_{k} W_{i k}(s) \\
& \times K_{k j}(s, u) d u d s .
\end{aligned}
$$

Equation (70) yields

$\left\|K^{(0)}(x, y)\right\| \leqslant \frac{1}{2} \int_{(x+y) / 2}^{\infty}\|\tilde{V}(s)\| d s=\frac{1}{2} \eta((x+y) / 2)$,

and

$$
\left\|K^{(1)}(x, y)\right\| \leqslant \frac{1}{2} \sigma((x+y) / 2) \xi(x),
$$

where we defined

$$
\xi(x)=\int_{x}^{\infty}\|W(s)\| s d s .
$$

By mathematical induction we obtain again

$$
\|K(x, y)\| \leqslant \frac{1}{2} \eta((x+y) / 2) \exp [\xi(x)],
$$

which provides the estimate

$$
D^{-1}(x) \| K(x, y) \leqslant \frac{1}{2} \exp (2 \beta|x|) \eta((x+y) / 2) \exp [\xi(x)] .
$$

Equations (72) and (62) are very similar; the main differences are the definitions of the involved nuclear potentials and the presence of the $\exp (2 \beta|x|)$ factor.

From Eqs. (63) and (72) it is seen that threshold energies have two consequences:

(a) The threshold energies appear explicitly in the translation kernel;

(b) An exponential decrease is required from the nuclear potentials, the measure of this decrease being expressed by

$$
4 \sup _{i, j}\left(\left|\lambda_{i}{ }^{2}-\lambda_{j}{ }^{2}\right|\right)^{1 / 2}
$$

Introduction of Coulomb forces in the scheme does not modify the method of solution, the Coulomb interaction being a scalar operator. Conditions similar to those of Ref. $6 \mathrm{~b}$ should be superimposed on the ones formulated in the present paper.

\section{APPENDIX A}

Let $A_{0}$ and $A$ be two partial differential matrix operators

$$
\begin{aligned}
& A \equiv \frac{d^{2}}{d x^{2}}-W_{0}(x)-V(x) \equiv \frac{d^{2}}{d x^{2}}-W(x), \\
& A_{0} \equiv \frac{d^{2}}{d x^{2}}-W_{0}(x) .
\end{aligned}
$$


Let now a transformation $X$ be defined by

$$
X f=I f+\int_{x}^{\infty} K(x, y) f(y) d y
$$

In Eq. (A2) $I$ is the identity matrix and $f$ and twice differential matrix satisfying

$$
\left\{\begin{array}{l}
\lim _{y \rightarrow \infty} f(y) \sim \exp (i k x) \\
k \text { real. }
\end{array}\right.
$$

The transformation $X$ is said to be a translation from $A_{0}$ to $A$ if for all $f$ 's one has

$$
A X f \equiv X A_{0} f .
$$

By integrations and derivations under the integral sign the kernel $K(x, y)$ of Eq. (A2) must satisfy the following Eq. (A4) for $X$ to exist. Equation (A4) is

$$
\begin{gathered}
\left(\frac{d^{2}}{d x^{2}}-W_{0}(x)-V(x)\right) K(x, y)=\frac{d^{2}}{d y^{2}} K(x, y)-K(x, y) W_{0}(y), \\
\lim _{y \rightarrow \infty} K(x, y)=\lim _{y \rightarrow \infty} \frac{\partial}{\partial y} K(x, y)=0, \\
K(x, x)=\frac{1}{2} \int_{x}^{\infty} V(s) d s .
\end{gathered}
$$

\section{APPENDIX B}

We consider the partial differential matrix equation

$$
\begin{aligned}
& \left(\frac{d^{2}}{d x^{2}}-\frac{d^{2}}{d y^{2}}\right) L(x, y)=V(s) L(x, y), \\
& L(x, x)=\frac{1}{2} \int_{x}^{\infty} W(s) d s \\
& \lim _{y \rightarrow \infty} L(x, y)=\lim _{y \rightarrow \infty} \frac{\partial}{\partial x} L(x, y)=0 .
\end{aligned}
$$

By the Riemann method Eq. (B1) is transformed into an integral equation:

$L(x, y)=\frac{1}{2} \int_{(x+y) / 2}^{\infty} V(s) d s+\frac{1}{2} \iint_{D} W(s) L(s, u) d u d s$.
Equation (B2) is a matrix equation similar to $\mathrm{Eq}$. (1.3.6) of Ref. 1. $D$ is the domain of Fig. (1). Therefore, one defines

$$
\begin{aligned}
& \eta(x)=\int_{x}^{\infty}\|V(s)\| d s, \\
& \xi(x)=\int_{x}^{\infty}\|W(s)\| d s,
\end{aligned}
$$

and obtains the estimate for the norm of $L(x, y)$,

$$
\|L(x, y)\| \leqslant \frac{1}{2} \eta((x+y) / 2) \exp [\xi(x)] .
$$

Equation (B4) gives sufficient conditions for the existence of a solution $L(x, y)$ for Eq. (B1).

\footnotetext{
*On sabbatical leave from the Department of Physics and Astronomy, University of Kentucky, Lexington, Kentucky, 40506.

${ }^{1} \mathrm{Z}$.S. Agranovitch and V.A. Marchenko, The Inverse Problem of Scattering Theory, translated by B.D. Seckler (Gordon and Breach, New York, 1963).

${ }^{2}$ For references for the one-dimensional problem: H. E. Moses, J. Math. Phys, 16, 1044 (1975).

${ }^{3}$ J. J. Loeffel, Ann. Inst. Henri Poincaré 8, (4), 339 (1968).

${ }^{4}$ P. J. Kelemen, J. Math. Phys. 12, 1142 (1971).

${ }^{5}$ H. I. Gugusvili and Y. L. Mentovski, Nuovo Cimento A 10 , 277 (1972)

${ }^{6}$ (a) M. Coz and C. Coudray, J. Math. Phys. 14, 1574 (1973);

(b) J. Math. Phys. (1976) to be published.

${ }^{7}$ M.M. Crum, Quart. J. Math. 6, (2), 121 (1955).

${ }^{8}$ M. G. Krein, Dokl. Akad. Nauk SSSR 113 (5), 970 (1957).

${ }^{9}$ R. G. Newton and R. Jost, Nuovo Cimento 1, 505 (1955).

${ }^{10}$ T. W. Chaundy, Quart. J. Math. Oxford 10, 310 (1939).

${ }^{11}$ E. T. Copson, Arch. Ratl. Mech. Anal. 1, 324 (1958).

${ }^{12} \mathrm{R}$. Courant and Hilbert, Methods of Mathematical Physics (Interscience, New York, 1943), Tח, p. 450.

${ }^{13}$ H. G. Garnir, M. de Wilde, and J. Schmets, Analyse fonctionnelle. 1 (Birkhauser Verlag, Bassel and Stuttgart, 1968 .

${ }^{14} \mathrm{G}$. Valiron, Equations fonctionnelles, Applications (Masson, Paris, 1950), p. 569; see also Ref.6b.
} 